\title{
Do we need an individual approach to atrial fibrillation and adrenergic overload in the critically ill?
}

\author{
MATIJA KALIŠNIK JURIJ ${ }^{1,2,3}$, MATEJ PODBREGAR ${ }^{1,4}$ \\ ${ }^{1}$ University of Ljubljana, Faculty of Medicine, Ljubljana, Slovenia \\ ${ }^{2}$ Department of Cardiovascular Surgery, University Medical Centre Ljubljana, Slovenia \\ ${ }^{3}$ Department of Cardiac Surgery, Cardiovascular Center, Klinikum Nuernberg-Paracelsus Medical University, Nuremberg, Germany \\ ${ }^{4}$ Clinical Department for Anaesthesiology and Surgical Intensive Care, University Medical Centre Ljubljana, Slovenia
}

Corresponding author:

Matej Podbregar

Cardiovascular Intensive Care Unit

Clinical Department for Anaesthesiology and Surgical Intensive Care

University Medical Centre Ljubljana

Zaloška 7, 1000 Ljubljana, Slovenia

E-mail:matej.podbregar@guest.arnes.si

\section{ABSTRACT}

Despite catecholamines being lifesaving drugs, they can also be harmful. Adrenergic overload is one of the major causes of supra- and ventricular arrhythmias, which induce haemodynamic instability of critically ill patients. In this paper we will focus on the pathophysiology of atrial fibrillation (AF), the importance of adrenergic overload for triggering AF, the importance of the autonomic nervous system and we will challenge the importance of decreasing adrenergic load with selective and nonselective $\beta$-blockers, which have different effects on the metabolism of the severely ill. We will also emphasize the importance of an individual approach due to pharmacogenetic differences in $\beta$-adrenergic signalling.

Key words: catecholamines, atrial fibrillation, beta-blockers, metabolism, resting energy expenditure

\section{INTRODUCTION}

Atrial fibrillation (AF) is a supraventricular tachycardia characterized electrically by chaotic atrial activation that results in mechanically ineffective atrial contraction. New-onset $\mathrm{AF}$ is a common arrhythmic complication of critical illness, with an incidence that varies from 4 to $9 \%$ in general intensive care unit patients, to 32 to $50 \%$ in patients after major cardiac and thoracic surgery. (1-2)

New-onset AF is associated with increased morbidity and mortality in patients hos- pitalized for heart failure, as well as various other critical conditions, although it is possible that AF in these cases is primarily a marker of disease severity rather than a direct cause of death. $(3,4)$

$\mathrm{AF}$ is associated with cardioembolic events and heart failure, longer hospital stays, and reduced quality of life as well as a two to five-fold increased mortality. (5-6)

AF in critically ill patients can present as asymptomatic ECG changes or, on the other hand, it can cause severe hemodynamic instability with profound hypotension, myocardial ischemia and heart failure leading to pulmonary edema, cardiogenic shock, with subsequent tissue hypoxia and organ dysfunction. Highly symptomatic patients are candidates for synchronized electro-cardioversion. (7)

Despite catecholamines being lifesaving drugs, they can also be harmful. Adrenergic overload is one of the major causes of hemodynamic instability due to supraand ventricular arrhythmias. In this paper we will focus on the pathophysiology of $\mathrm{AF}$, the importance of adrenergic overload for triggering AF, the importance of the autonomic nervous system and in the end we will challenge the importance of decreasing adrenergic load. We will also emphasize the importance of an individual approach due to pharmacogenetic differences in $\beta$-adrenergic signaling. The catecholamines also have other non-cardiovascular effects, i.e. they have profound metabolic effects, elevating resting energy expenditure and changing substrate oxidation rates. Finally, we will discuss the possible pathways and effects of selective and non-selective $\beta$-blockers on the hyper- metabolism of the severely ill.

\section{PATHOPHYSIOLOGY OF AF}

Structural and electrical atrial remodeling are fundamental mechanisms for AF. (8) The majority of critically ill patients have already acquired some structural and electrical atrial remodeling before intensive care unit (ICU) admission. Structural remodeling, particularly fibrosis, is the mainstay in many forms of AF. This fibrosis is primarily due to atrial dilatation, which leads to the local activation of the renin aldosterone angiotensin system (RAAS) and further initiates multiple cell signaling cascades, including inflammation and apoptosis, stimulating fibrosis, as well as possible modulation of ion channels and gap-junction dynamics. (9) Fibroblasts can couple electrically to cardiomyocytes and when increased in number, promote reentry and/or ectopic activity. (10)

Electrical remodeling promotes AF by acting on the fundamental arrhythmia mechanism: focal ectopic activity and reentry. In this context, two principles gain attention: factors triggering the onset and factors perpetuating AF. (11)

Ectopic focal discharges often initiate AF. Rapidly firing foci initiating paroxysmal $\mathrm{AF}$ arise most commonly from the atrial myocardial sleeves that extend into pulmonary veins. (11) Although the pulmonary veins are the most common sites for ectopic focal triggers, they can also arise 
elsewhere, including the posterior LA (left atrium), ligament of Marshall, coronary sinus, venae cavae, septum, and appendages. Atrial myocardial fibers are oriented in disparate directions, and possess unique anatomical and electrophysiological features for their arrhythmogenic nature. The relatively depolarized resting potentials in pulmonary vein myocytes promote sodium channel inactivation leading to abrupt changes in fiber orientation and thus favouring reentry. These myocytes also demonstrate abnormal automaticity and triggered activity that could promote rapid focal firing. (11)

The evolution of AF from paroxysmal to persistent to permanent forms through atrial remodeling can be caused by the arrhythmia itself and/or progression of underlying heart disease. Atrial electrical properties are modified by affecting expression and function of ion-channels, pumps, and exchangers, thus a reentry prone substrate is created which promotes arrhythmia. This concept is known as atrial remodeling and was first tested in animal models showing that long-term rapid atrial pacing or maintenance of AF favors the occurrence and maintenance of $\mathrm{AF}$ ('AF Begets AF'). (12) The developments of functional reentry substrates, which are reversible on AF termination, contribute to persistent AF.

There are more potential mechanisms for ectopic triggering. The resting potential of a normal atrial cell is maintained by high resting $\mathrm{K}+$ permeability through the inward rectifier K+ current (IK1). Although normal human atrial cells also manifest pacemaker current (If), it is overwhelmed by much larger IK1, and does not manifest automaticity. Enhanced automaticity is caused by changes in this balance resulting from decreased (IK1) and/ or enhanced (If). (13)

Early after depolarizations (EAD) involve abnormal secondary cell membrane depolarization during repolarization phases. EAD are caused mainly by action potential duration prolongation (i.e. congenital long QT-Syndrome). (14) This allows L-type $\mathrm{Ca} 2+$ current (ICaL) to recover from inactivation, leading to inward movement of $\mathrm{Ca} 2+$ ions causing EAD.

Delayed after depolarizations (DAD) are caused by abnormal diastolic release of $\mathrm{Ca} 2+$ from sarcoplasmic reticulum stores. Specialized sarcoplasmic reticulum $\mathrm{Ca} 2+$ channels (called ryanodine receptors
[RyRs]) release $\mathrm{Ca} 2+$ in response to transmembrane Ca2+ entry. (11) RyRs are normally closed during diastole but can open if they are functionally defective or if the sarcoplasmic reticulum is $\mathrm{Ca} 2+$ overloaded. When one $\mathrm{Ca} 2+$ ion is released during diastole, it is exchanged for three extracellular $\mathrm{Na}+$ ions by the $\mathrm{Na}+-\mathrm{Ca} 2+$ exchanger, causing a net depolarizing inward positive-ion movement (called transient inward current [Iti]) that underlies DADs. Congestive heart failure, one of the most common causes of AF, produces atrial cell $\mathrm{Ca} 2+$ overload and DADs. (15)

\section{EFFECTS OF THE AUTONOMIC NERV- OUS SYSTEM}

Parasympathetic stimulation causes vagal discharge which enhances acetylcholine dependent $\mathrm{K}+$ current (IKACh), reducing atrial action potential duration and refractoriness, increasing the susceptibility to reentry mechanism. (11) Sympathetic stimulation causes $\beta$-adrenoceptor (AR) activation, which increases diastolic $\mathrm{Ca} 2+$ leak and promotes DAD by hyperphosphorylating RyR2s, which promotes automaticity and triggered activity. Atrial sympathetic hyperinnervation

occurs in persistent AF patients. (16) Autonomic neural remodeling contributes to positive feedback loops that promote AF persistence and recurrence. Plexi of autonomic ganglia that constitute the intrinsic cardiac autonomic nervous system are located in epicardial fat near the pulmonary vein-LA junctions, at the orifices of venae cavae in the right atrium and the ligament of Marshall. AF studies in critically ill patients after cardiac surgery have demonstrated that at least two routes of cardiac autonomic modulation pave the way to AF, (17-18) whereby a landmark study reported concomitant vagal withdrawal and sympathetic activation as a mode of perioperative AF activation. In sharp contrast, we have shown that patients developing AF after cardiac surgery, having had been on complete chronic beta blockade, exhibit different, parasympathetically (co)mediated routes of cardiac autonomic modulation with concomitant parasympathetic and excessive adrenergic activation. (19)

\section{PROMOTORS OF AF IN CRITICALLY ILL PATIENTS}

In critically ill patients with AF, we can detect and modify promotors of AF. (20) The most important promotor is adrenergic overstimulation (i.e. stress, pain and inotropic support). Other important promoters are myocardial (atrial) stretch (i.e. fluid overload, acute mitral regurgitation, mitral stenosis, pulmonary embolism), inappropriate oxygen delivery to the myocardium (i.e. myocardial ischemia, hypovolemia, an1mia), electrolyte disturbance (i.e. hypokalemia, hypomagnesemia), systemic and local inflammation (i.e. after on-pump cardiac surgery, sepsis, myo/pericarditis), hypothermia, concomitant increased vagal activity and intrinsic cardiac autonomous system hyperreactivity and endocrine disorders (i.e. hyperthyroidism, pheochromocytoma).

\section{ADRENERGIC OVERLOAD}

A recently published review explores the schizophrenic 'Jekyll-and-Hyde' characteristics of catecholamines in critical illness, as they are both necessary for survival yet detrimental in excess. (21) A hyperadrenergic state is responsible for the reversible myocardial depression seen in both phaeochromocytoma crisis (22) and the stress-related ("broken heart", Takotsubo) cardiomyopathy. (23) Adrenergic overstimulation is associated with a poor prognosis in acute coronary syndromes, heart failure, liver cirrhosis and acute cerebrovascular disease. (24-25)

Despite association with adverse outcomes, adrenergic agonists remain the cornerstone of cardiovascular support. Norepinephrine is the current recommended first-line agent for low vascular resistance states, while dobutamine is recommended for myocardial dysfunction. (26) Epinephrine has both inotropic and pressor properties that can be used as an alternative to either. (27) It is likely that these exogenous catecholamines will add further to the endogenous stress response, therefore increasing total adrenergic stress. (21) It was shown that dobutamine administration was independently associated with increased mortality in acute heart failure and after cardiac surgery. $(28,29)$ High levels of catecholamines as well as a persistently high heart rate predict poor patient outcomes in sepsis. $(30,31)$ While high catecholamine levels could simply be a marker of disease severity, they may also be a perpetrator of further organ dysfunction. (21) Increasing catecholamine doses were associated with increasing mortality, independent of effects on blood pressure. (32)

One of the steps towards reducing adrenergic overload is to not necessarily target 
"normal" or "supranormal" haemodynamic values. (21) While too low a blood pressure or cardiac output may compromise tissue perfusion and oxygenation, neither increasing blood pressure $>65 \mathrm{mmHg}(34)$ nor targeting "supranormal" values of cardiac output (34) translated into an overall survival benefit. Previously normotensive patients trended to worse outcomes when a higher blood pressure was targeted. (34) Unrecognized diastolic dysfunction may be also compromised further by the use of catecholamines. (35)

\section{PHARMACOGENETICS}

In some patients, inappropriate high ventricular rate is noticed despite relatively low inotropic or vasopressor dose after adequate volume resuscitation.

Pharmacogenetics can provide an answer to this diversity. (36) Twelve singlenucleotide polymorphisms have been identified in the $\beta 1-A R$, but only two of these are thought to be clinically relevant. At position 389, the glycine nucleotide in the G-protein coupling domain can be substituted for arginine. (37) This is again of function polymorphism, resulting in increased adenylate cyclase activity. The Arg/Arg genotype is associated with increased sensitivity of the $\beta 1-A R$ to noradrenaline, (38) a 3- to 4-fold increase in signal transduction and an increase in the number of constitutionally active receptors compared with the Arg/Gly or Gly/ Gly genotypes. (39) The other important $\beta 1$-AR polymorphism is at position 49 and is thought to have a modulating role in adenylate cyclase activity. (39) The gain of function Arg/Arg polymorphism is important because higher adrenergic activity has been shown to increase the likelihood of $\mathrm{AF}$ induction in a dose-dependent manner. (40) Bucindolol, a competitive antagonist of the $\beta 1-\mathrm{AR}$, facilitates the inactivation of constitutionally active receptors (inverse agonism), and decreases levels of noradrenaline. (43) Bucindolol prevented new-onset AF in patients with heart failure with reduced ejection fraction in $74 \%$ of patients with the Arg/Arg genotype, but had no effect in those patients with the Gly/Gly genotype. (41) The substudy found that all-cause and cardiovascular mortality, as well as cardiovascular and heart failure hospitalizations were significantly reduced in patients with the Arg/Arg genotype, but not glycine carriers. (42) The enhanced adrenergic signaling in the Arg/ Arg genotype may render it more susceptible to $\beta$ blocking drugs sympatholytic actions, thereby preventing the induction of AF that might normally occur in these patients. Interestingly, the loss of function glycine 389 polymorphism is associated with a significantly better response to rate-controlling therapies in patients with AF. (43) This may be explained because the rate-control therapies can work synergistically with the attenuated $\beta 1$-adrenergic cascade caused by this genotype.

$\beta 1$-AR polymorphisms could also influence the efficacy of amiodarone because it possesses antiadrenergic effects. (44)

\section{B-ANTAGONISTS AND DECATECHO- LAMINIZATION IN CRITICALLY ILL PATIENTS}

According to current guidelines, $\beta$-adrenergic blockade is the first line treatment of AF in patients with preserved left ventricular function, and $\beta$-adrenergic blockade should also be considered in patients with decreased left ventricular function. $(45,46)$ Landiolol, an ultra- short acting $\beta$-antagonist, seems to be fast, effective and safe in converting AF to sinus rhythm in post- operative cardiac surgery patients. (47) At low dose, landiolol facilitates a high rate of conversion to sinus rhythm (69\%) in patients with sepsis and supraventricular tachycardia without haemodynamic deterioration. (48)

In a poor prognosis subset of patients with septic shock, i.e. requiring high doses of catecholamines after $24 \mathrm{~h}$ and with concurrent tachycardia, esmolol demonstrated significant reductions in mortality, time on vasopressors, and renal and myocardial injury compared to the control group. (49) Further studies should confirm the data from this revolutionary idea. In our opinion, the extreme caution in patient selection based on echocardiografically determined preserved systolic and impaired diastolic left/right ventricular function and very low initial dose of ultra-selective $\beta 1$-blocker is necessary so as not to induce harm to the critically ill septic patient. (50) In the future, it will probably be important even to determine the pharmacogenetic profile of $\beta$-AR in these patients.

Critical illness and management in a critical care unit are characterised by a severe and abnormally prolonged stressor response; this response may become maladaptive. (21) Given this premise, attenuation of an excessive adrenergic component of the stress reaction is a tempting therapeutic option during sepsis and other critically ill states.
Titration of $\beta$-blocker dosing to a target heart rate appears feasible without compromising haemodynamics in most patients; stroke volume usually increases while catecholamine requirements decrease. (51) Possible mechanisms include improved ventricular filling and ventricular-arterial coupling; restoration of adrenergic receptor density, which may have been reduced by excessive catecholamine stimulation; $(52,53)$ and a decrease in the systemic inflammatory response. (54)

Patient selection and close monitoring are likely to be crucial in this setting because of the risk of worsening myocardial dysfunction. (21)

The pharmacogenetic properties of $\beta$-blockers and an individual approach are, therefore, an important area for further research to further understand which critically ill patients will benefit from both existing and novel therapies for AF and supraventricular tachycardia.

\section{METABOLIC EFFECTS OF B-BLOCKERS}

The majority of critically ill patients have a high resting energy expenditure (REE); this is especially true for patients with burns, after severe trauma and in sepsis. (55) Also, patients with heart failure, who are not cachectic, have high REE. $(56,57)$ It has been demonstrated that selective and nonselective $\beta$-blockers reduce the REE. (58) Nonselective $\beta$-blockers appear to shift total body substrate use from fatty-acid to glucose oxidation. (58-59) As less oxygen is needed for the oxidation of glucose than for the oxidation of fatty acids, (60) this as a favorable effect on myocardial oxygen demand in heart failure. The molecular mechanisms by which nonselective $\beta$-blockers promote glucose oxidation are not known, but it has been demonstrated in mice that the receptor NOR-1, which is a target of $\beta$-adrenergic signaling, regulates expression of genes that encode proteins that control oxidative metabolism, such as PGC-1 $\alpha$, lipin-1 $\alpha$, FOXO1, and the enzyme pyruvate dehydrogenase kinase type 4 (PDK4). (61) This last, PDK4, is an isoform of PDK that is directly involved in the regulation of the entry of glycolysis products into oxidative metabolism. This is also one possible explanation why only the nonselective $\beta$-blockers appear to influence the shift of metabolism to glucose oxidation - because they do not only interact with the target $\beta 1$-adrenergic receptors. Clinical studies have confirmed the metabolic ef- 
fects of non-selective blockers, such as propranolol, on reduced hypermetabolism in burns, which could be prolonged up to 2 years, and carvedilol, which attenuated the development and promoted a partial reversal of cachexia in patients with severe chronic heart failure, supporting a role for prolonged sympathetic activation in the genesis of weight loss. $(62,63)$

\section{CONCLUSIONS}

Catecholamine administration is useful and even life-saving for short-term restoration of tissue perfusion or correction of life threatening hypotension. However, catecholamines are poisonous when given in excess, causing regional ischemia, triggering arrhythmia and promoting systemic inflammation. Individual titration of short-acting, selective $\beta-1$ blockers, seems to be a promising approach to supraventricular tachycardia and to maladaptive response to sepsis, especially in the haemodynamically stable phase of disease. On the other hand, non-selective $\beta$-blockers are important regulators of whole body metabolism, capable of reducing resting energy expenditure, attenuating the development and promoting a partial reversal of cachexia.

\section{REFERENCES}

1. Seguin P, Launey Y. Atrial fibrillation is not just an artefact in the ICU. Crit Care 2010; 14:182.

2. Ivanovic J, Maziak DE, Ramzan S, McGuire AL, Villeneuve PJ, Gilbert S, et al. Incidence, severity and perioperative risk factors for atrial fibrillation following pulmonary resection. Interact Cardiovasc Thorac Surg 2014 Mar;18(3):340-6.

3. Maisel WH, Rawn JD, Stevenson WG. Atrial fibrillation after cardiac surgery. Ann Intern Med 2001;135:1061-73.

4. Walsh SR, Tang T, Gaunt ME, Schneider HJ. New arrhythmias after non-cardiothoracic surgery. BMJ 2006;333:715.

5. Walkey AJ, Wiener RS, Ghobrial JM, Curtis LH, Benjamin EJ. Incident stroke and mortality associated with new-onset atrial fibrillation in patients hospitalized with severe sepsis. JAMA 2011 Nov 23;306(20):2248-54.

6. Auer J, Weber T, Berent R, Ng CK, Lamm G, Eber B. Risk factors of postoperative atrial fibrillation after cardiac surgery. J Card Surg 2005 Sep-Oct;20(5):425-31.

7. Marinsek M, Larkin GL, Zohar P, Bervar M, Pekolj-Bicanic M, Mocnik FS, et al. Efficacy and impact of monophasic versus biphasic countershocks for transthoracic cardioversion of persistent atrial fibrillation. Am J Cardiol 2003 Oct 15;92(8):988-91.

8. Nabar A, Pathan I. Pathophysiology of Atrial Fibrillation-current Concepts. J Assoc Physicians India 2016 Aug;64(8):11-5.

9. Healey JS, Baranchuk A, Crystal E. Prevention of atrial fibrillation with angiotensin converting enzyme inhibitors and angiotensin receptor blockers: a meta-analysis. J Am Coll Cardiol 2005;45:1832-41.

10. Yue L, Xie J, Nattel S. Molecular determinants of cardiac fibroblast electrical function and therapeutic implications for atrial fibrillation. Cardiovasc Res 2011;89:744-53.

11.lich JR, Cha TJ, Zhang L. Cellular electrophysiology of canine pulmonary vein cardiomyocytes: action potential and ionic current properties. J Physiol 2003;551:801-4.

12. Wijffels MC, Kirchhof CJ, Dorland R, Allessie MA. Atrial fibrillation begets atrial fibrillation. A study in awake chronically instrumented Varro A, Cerbai E goats. Circulation 1995;92:1954-2022.

13. Stillitano F, Lonardo G, Zicha S, , Mugelli A, Natell S. Molecular basis of funny current (If ) in normal and failing human heart. J Mol Cell Cardiol 2008;45:289-99.

14. Johnson JN, Tester DJ, Perry J, Salisbury BA, Reed CR, Ackerman MJ. Prevalence of early-onset atrial fibrillation in congenital long QT syndrome. Heart Rhythm 2008;5:704-9.

15. Yeh YH, Wakili R, Qi XY, Chartier D, Boknik P, Kääb S, et al. Calcium-handling abnormalities underlying atrial arrhythmogenesis and contractile dysfunction in dogs with congestive heart failure. Circ Arrhythm Electrophysiol 2008 Jun 1;1(2):93-102.

16. Chang CM, Wu TJ, Zhou S, Doshi RN, Lee MH, Ohara T, et al. Nerve sprouting and sympathetic hyperinnervation in a canine model of atrial fibrillation produced by prolonged right atrial pacing. Circulation 2001 Jan 2;103(1):22-5.

17. Aurora R. Recent insights into the role of the autonomic nervous system in the creation of substrate for atrial fibrillation - Implications for therapies targeting the atrial autonomic nervous system. Circ Arrhythm Electrophysiol 2012;5:850-9.

18. Amar D, Zhang H, Miodownik S. Competing autonomic mechanisms precede the onset of postoperative atrial fibrillation. J Am Coll Cardiol 2003;7:1262-8.

19. Kališnik JM, Hrovat E, Hrastovec A, Avbelj V, Žibert J, Geršak B. Severe cardiac autonomic derangement and altered ventricular repolarization pave the way to postoperative atrial fibrillation. Innovations 2015;10:398-405.

20. Arrigo M, Bettex D, Rudiger A. Management of atrial fibrillation in criticallyill patients. Crit Care Res Pract 2014;2014:840615.

21. Andreis DT, Singer M. Catecholamines for inflammatory shock: a Jekyll-and-Hyde conundrum. Intensive Care Med 2016 Sep;42(9):1387-97.

22. Farrugia FA, Martikos G, Tzanetis P, Charalampopoulos A, Misiakos E, Zavras N,Sotiropoulos D. Pheochromocytoma, diagnosis and treatment: Review of the literature. Endocr Regul 2017 Jul 1;51(3):168-81.

23. Dawson DK. Acute stress-induced (takotsubo) cardiomyopathy. Heart. 2017 Aug 20. pii: heartjnl-2017-311579. doi: 10.1136/ heartjnl-2017-311579.

24. Ostrowski SR, Pedersen SH, Jensen JS, Mogelvang R, Johansson PI. Acute myocardial infarction is associated with endothelial glycocalyx and cell damage and a parallel increase in circulating catecholamines. Crit Care 2013 Feb 22;17(1):R32.

25. Feibel JH, Hardy PM, Campbell RG, Goldstein MN, Joynt RJ. Prognostic value of the stress response following stroke. JAMA 1977 Sep 26;238(13):1374-6.

26. Rhodes A, Evans LE, Alhazzani W, Levy MM, Antonelli M, Ferrer R, et al. Surviving Sepsis Campaign: International Guidelines for Management of Sepsis and Septic Shock:2016. Intensive Care Med 2017 Mar;43(3):304-77.

27. Myburgh JA, Higgins A, Jovanovska A, Lipman J, Ramakrishnan N, Santamaria J;CAT Study investigators. A comparison of epineph- 
rine and norepinephrine in critically ill patients. Intensive Care Med 2008 Dec;34(12):2226-34.

28. Abraham WT, Adams KF, Fonarow GC, Costanzo MR, Berkowitz RL, LeJemtel TH, et al; ADHERE Scientific Advisory Committee and Investigators; ADHERE Study Group. In-hospital mortality in patients with acute decompensated heart failure requiring intravenous vasoactive medications: an analysis from the Acute Decompensated Heart Failure National Registry (ADHERE). J Am Coll Cardiol 2005 Jul 5;46(1):57-64.

29. Shahin J, DeVarennes B, Tse CW, Amarica DA, Dial S. The relationship between inotrope exposure, six-hour postoperative physiological variables, hospital mortality and renal dysfunction in patients undergoing cardiac surgery. Crit Care 2011 Jul 7;15(4):R162.

30. Brown SM, Lanspa MJ, Jones JP, Kuttler KG, Li Y, Carlson R, et al. Survival after shock requiring high-dose vasopressor therapy. Chest 2013 Mar;143(3):664-71.

31. Leibovici L, Gafter-Gvili A, Paul M, Almanasreh N, Tacconelli E, Andreassen S,et al; TREAT Study Group. Relative tachycardia in patients with sepsis: an independent risk factor for mortality. QJM 2007 Oct;100(10):629-34.

32. Dünser MW, Ruokonen E, Pettilä V, Ulmer H, Torgersen C, Schmittinger CA, et al. Association of arterial blood pressure and vasopressor load with septic shock mortality: a post hoc analysis of a multicenter trial. Crit Care 2009;13(6):R181.

33. Asfar P, Meziani F, Hamel JF, Grelon F, Megarbane B, Anguel N, et al; SEPSISPAM Investigators. High versus low blood-pressure target in patients with septic shock. N Engl J Med 2014 Apr 24;370(17):1583-93.

34. Gattinoni L, Brazzi L, Pelosi P, Latini R, Tognoni G, Pesenti A, Fumagalli R. A trial of goal-oriented hemodynamic therapy in critically ill patients. SvO2 Collaborative Group. N Engl J Med 1995 Oct 19;333(16):1025-32.

35. Landesberg G, Gilon D, Meroz Y, Georgieva M, Levin PD, Goodman S, et al. Diastolic dysfunction and mortality in severe sepsis and septic shock. Eur Heart J 2012 Apr;33(7):895-903.

36. Hanley CM, Robinson VM, Kowey PR. Status of Antiarrhythmic Drug Development for Atrial Fibrillation: New Drugs and New Molecular Mechanisms. Circ Arrhythm Electrophysiol 2016 Mar;9(3):e002479.

37. Nia AM, Caglayan E, Gassanov N, Zimmermann T, Aslan O, Hellmich M, et al. Beta1-adrenoceptor polymorphism predicts flecainide action in patients with atrial fibrillation. PLoS One $2010 \mathrm{Jul}$ 2;5(7):e11421.

38. O'Connor CM, Fiuzat M, Carson PE, Anand IS, Plehn JF, Gottlieb SS, et al. Combinatorial pharmacogenetic interactions of bucindolol and $\beta 1, \alpha 2 \mathrm{C}$ adrenergic receptor polymorphisms. PLoS One 2012;7(10):e44324.

39. Liggett SB, Mialet-Perez J, Thaneemit-Chen S, Weber SA, Greene SM, Hodne D, et al. A polymorphism within a conserved beta(1)adrenergic receptor motif alters cardiac function and beta-blocker response in human heart failure. Proc Natl Acad Sci U S A 2006 Jul 25;103(30):11288-93.

40. Oral H, Crawford T, Frederick M, Gadeela N, Wimmer A, Dey S, et al. Inducibility of paroxysmal atrial fibrillation by isoproterenol and its relation to the mode of onset of atrial fibrillation. J Cardiovasc Electrophysiol 2008;19:466-70.

41. Aleong RG, Sauer WH, Davis G, Murphy GA, Port JD, Anand IS, et al. Prevention of atrial fibrillation by bucindolol is dependent on the beta 389 Arg/Gly adrenergic receptor polymorphism. JACC Heart Fail 2013 Aug;1(4):338-44.

42. Kao DP, Davis G, Aleong R, O’Connor CM, Fiuzat M, Carson PE, et al. Effect of bucindolol on heart failure outcomes and heart rate response in patients with reduced ejection fraction heart failure and atrial fibrillation. Eur J Heart Fail 2013 Mar;15(3):324-33.

43. Parvez B, Chopra N, Rowan S, Vaglio JC, Muhammad R, Roden DM, Darbar D. A common $\beta 1$-adrenergic receptor polymorphism predicts favorable response to rate-control therapy in atrial fibrillation. J Am Coll Cardiol 2012;59:49-56.

44. Lalevée N, Nargeot J, Barrére-Lemaire S, Gautier P, Richard S. Effects of amiodarone and dronedarone on voltage-dependent sodium current in human cardiomyocytes. J Cardiovasc Electrophysiol 2003;14:885-90.

45. Kirchhof P, Benussi S, Kotecha D, Ahlsson A, Atar D, Casadei B, et al. 2016 ESC Guidelines for the management of atrial fibrillation developed in collaboration with EACTS. Europace 2016 Nov;18(11):1609-78.

46. Andrade JG, Macle L, Nattel S, Verma A, Cairns J. Contemporary Atrial Fibrillation Management: A Comparison of the Current AHA/ACC/HRS, CCS, and ESC Guidelines. Can J Cardiol 2017 Aug;33(8):965-76.

47. Shibata SC, Uchiyama A, Ohta N, Fujino Y. Efficacy and Safety of Landiolol Compared to Amiodarone for the Management of Postoperative Atrial Fibrillation in Intensive Care Patients. J Cardiothorac Vasc Anesth 2016 Apr;30(2):418-22.

48. Okajima M, Takamura M, Taniguchi T. Landiolol, an ultra-short-acting $\beta 1$-blocker, is useful for managing supraventricular tachyarrhythmias in sepsis. World J Crit Care Med 2015 Aug 4;4(3):251-7.

49. Morelli A, Ertmer C, Westphal M, Rehberg S, Kampmeier T, Ligges S, et al. Effect of heart rate control with esmolol on hemodynamic and clinical outcomes in patients with septic shock: a randomized clinical trial. JAMA 2013 Oct 23;310(16):1683-91.

50. Ikeshita K, Nishikawa K, Toriyama S, Yamashita T, Tani Y, Yamada T, Asada A. Landiolol has a less potent negative inotropic effect than esmolol in isolated rabbit hearts. J Anesth 2008;22(4):361-6.

51. Morelli A, Donati A, Ertmer C, Rehberg S, Kampmeier T, Orecchioni A, et al. Microvascular effects of heart rate control with esmolol in patients with septic shock: a pilot study. Crit Care Med 2013 Sep;41(9):2162-8.

52. Suzuki T, Morisaki H, Serita R, Yamamoto M, Kotake Y, Ishizaka A, Takeda J. Infusion of the beta-adrenergic blocker esmolol attenuates myocardial dysfunction in septic rats. Crit Care Med 2005 Oct;33(10):2294-301.

53. Heilbrunn SM, Shah P, Bristow MR, Valantine HA, Ginsburg R, Fowler MB. Increased beta-receptor density and improved hemodynamic response to catecholamine stimulation during long-term metoprolol therapy in heart failure from dilated cardiomyopathy. Circulation 1989 Mar;79(3):483-90.

54. Gore DC, Wolfe RR. Hemodynamic and metabolic effects of selective beta1 adrenergic blockade during sepsis. Surgery 2006 May;139(5):686-94.

55.Zusman O, Theilla M, Cohen J, Kagan I, Bendavid I, Singer P. Resting energy expenditure, calorie and protein consumption in critically ill patients: a retrospective cohort study. Critical Care 2016;20:367.

56. Podbregar M, Voga G. Effect of selective and nonselective $\beta$-blockers on resting energy production rate and total body substrate utilization in chronic heart failure. J Card Fail 2002;8:369-78. 
57. Lainscak M, Podbregar M, Anker SD. How does cachexia influence survival in cancer, heart failure and other chronic diseases? Curr Opin Support Palliat Care 2007 Dec;1(4):299-305.

58. Lamont LS, Romito RA, Finkelhor RS, Kalhan SC. $\beta$-1-Adrenoreceptors regulate resting metabolic rate. Med Sci Sports Exerc 1997;29(6):769-74.

59. Kovacic D, Marinsek M, Gobec L, Lainscak M, Podbregar M. Effect of selective and non-selective beta-blockers on body weight, insulin resistance and leptin concentration in chronic heart failure. Clin Res Cardiol 2008 Jan;97(1):24-31.

60. Salamon S, Podbregar E, Kubatka P, Büsselberg D, Caprnda M, Opatrilova R, et al. Glucose Metabolism in Cancer and Ischemia: Possible Therapeutic Consequences of the Warburg Effect. Nutr Cancer 2017 Feb-Mar;69(2):177-83.

61. Pearen MA, Myers SA, Raichur S, Ryall JG, Lynch GS, Muscat GEO. The orphan nuclear receptor, NOR-1, a target of $\beta$-adrenergic signaling, regulates gene expression that controls oxidative metabolism in skeletal muscle. Endocrinology 2008;149(6):2853-65.

62. Wischmeyer PE, San-Millan I. Winning the war against ICU-acquired weakness: new innovations in nutrition and exercise physiology. Critical Care 2015;19(Suppl 3):S6. doi:10.1186/cc14724.

63. Clark AJS, Coats HK, Katus HA, Mohacsi P, Salekin D, Schultz MK, et al. Effect of beta-adrenergic blockade with carvedilol on cachexia in severe chronic heart failure: results from the COPERNICUS trial Andrew L. Anker J Cachexia Sarcopenia Muscle 2017 Aug;8(4):549-56. 